\title{
Infarto agudo do miocárdio e injeção intramiocárdica experimental em cães: estudos clínico, enzimático, eletrocardiográfico e ecocardiográfico
}

\author{
[Experimental acute myocardial infarction in dogs: clinic, enzymatic, electrocardiographic \\ and echocardiographic studies] \\ G.A.O. Cavalcanti ${ }^{1}$, R.B. Araújo ${ }^{2 *}$, E.G. Melo ${ }^{2}$, M.C.V. Moreira ${ }^{3}$, K.B.G. Borges ${ }^{4}$, \\ M.M. Melo ${ }^{2}$, L.R. Borboleta ${ }^{1}$, R.A.L. Muzzi $i^{5}$ \\ ${ }^{1}$ Aluno de pós-graduação - Escola de Veterinária - Universidade Federal de Minas Gerais - Belo Horizonte, MG \\ ${ }^{2}$ Escola de Veterinária - Universidade Federal de Minas Gerais - Belo Horizonte, MG \\ ${ }^{3}$ Faculdade de Medicina - Universidade Federal de Minas Gerais - Belo Horizonte, MG \\ ${ }^{4}$ Colégio Técnico - Universidade Federal de Minas Gerais - Belo Horizonte, MG \\ ${ }^{5}$ Universidade Federal de Lavras - Lavras, MG
}

\begin{abstract}
RESUMO
Os eventos isquêmicos em cães são incomuns, porém podem estar sendo subnotificados. Avaliou-se o infarto agudo do miocárdio (IAM) clinicamente, por meio de eletrocardiografia (ECG), eletrocardiografia contínua (EC), ecocardiografia (ECO), enzima creatina quinase (CK), enzima creatina quinase fração MB (CK-MB) e anátomo-histologicamente em cães sem raça definida, e observou-se a ocorrência de arritmias após injeção intramiocárdia por EC. O IAM foi obtido após a ligadura da coronária descendente anterior. Os animais apresentaram ao ECO dilatação da câmara esquerda e aumento do índice de desempenho miocárdico. Ao ECG houve desnivelamento de ST nas derivações pré-cordiais V1 e V2. No EC observaram-se arritmias ventriculares graves e supradesnivelamento de ST. As enzimas CK e CK-MB aumentaram significativamente, sendo que os picos de CK-MB e de CK ocorreram seis horas e 12 horas, respectivamente, após o IAM. Na análise histológica constatou-se infarto da parede inferior do ventrículo esquerdo e substituição do tecido muscular por tecido fibroso. Avaliou-se a injeção intramiocárdica por EC que pode servir como via terapêutica cardíaca, não sendo observado aumento das arritmias ventriculares após a injeção no miocárdio infartado. $\mathrm{O}$ infarto em cães pode ser detectado pelos exames cardíacos disponíveis, e a injeção intramiocárdica é uma via terapêutica cardíaca possível.
\end{abstract}

Palavras-chaves: cão, infarto do miocárdio, canídeos, eletrocardiografia contínua, creatina quinase, injeção intramiocárdica

\begin{abstract}
Ischemic events in dogs are uncommon; however, this may be under-reported. The myocardial infarction was created by left anterior descending coronary ligation in healthy mongrel dogs in clinical and laboratorial exams. These dogs were evaluated clinically, electrocardiography (ECG), through ambulatory electrocardiography $(A E)$, echocardiography (ECO), creatine kinase enzyme $(C K)$, creatine kinase $M B$ fraction enzyme $(C K-M B)$ and histopathologically. Even in these animals we observed the occurrence of arrhythmia after intramyocardial injection by AE. The animals exhibited left ventricular chamber enlargement and increase in myocardial performance index at ECO. In ECG, there were deviations in ST segment in the precordial leads VI and V2. CK and CK-MB showed high increase, CK and CK-MB peaks occurred six and 12 hours after infarction, respectively. Histopathology of the infarction in the inferior wall of the left ventricle and replacement of muscle tissue by fibrous tissue were seen. Furthermore, intramyocardial injection that may be used for therapeutic purposes was evaluated by AE, which demonstrated no increase in the ventricular arrhythmias. Therefore, myocardial infarction in dogs can be detected with the tests available and intramyocardial injection can be used as a therapeutic way.
\end{abstract}

Keywords: dog, myocardial infarct, canine, ambulatory electrocardiography, creatine kinase, myocardial injection

Recebido em 27 de setembro de 2010

Aceito em 23 de dezembro de 2011

*Autor para correspondência (corresponding author)

E-mail: baracat@vet.ufmg.br 


\section{INTRODUÇÃO}

O infarto miocárdico (IM) é a principal causa de morte em seres humanos devido às arritmias no infarto agudo (IAM) ou ao remodelamento cardíaco, causando, muitas vezes, insuficiência cardíaca congestiva (ICC) por dilatação cardíaca, a despeito do arsenal terapêutico disponível. Portanto, novas alternativas têm sido pesquisadas com terapia celular ou terapia com fatores de crescimento via injeção intramiocárdica ou intracoronária, com a finalidade de se minimizar os efeitos de tal remodelamento (Hubka et al., 2006; Leri et al., 2007).

O IAM é bem caracterizado nos seres humanos, principalmente pelos métodos diagnósticos eletrocardiograma (ECG), enzimático, ecocardiograma (ECO) e por eletrocardiografia contínua (EC) (Topol et al., 1998). Em cães, o IAM tem sido relatado (Falk e Jönsson, 2000; Almeida et al., 2007; Ware, 2007), porém nenhum estudo completo foi encontrado no auxílio diagnóstico; além disso, mesmo já tendo sido realizada a técnica de injeção intramiocárdica em coelhos (Zhang et al., 2007), ratos (Li et al., 2011) e em suínos, a avaliação eletrocardíaca mais profunda só foi realizada na espécie suína (Tondato et al., 2011).

Descreve-se que cães de grande porte com dilatação cardíaca e pouca diminuição dos índices sistólicos ao ECO podem estar sendo mal diagnosticados como portadores de cardiomiopatia dilatada (CD) quando, na verdade, apresentam arteriosclerose e pequenas áreas isquêmicas no miocárdio (Falk e Jönsson, 2000). Já a aterosclerose coronariana - comum em seres humanos - é uma condição rara em cães, mas pode ocorrer associada a casos de estenose aórtica, hipotireoidismo, hiperlipidemia e hipercolesterolemia (Lindsay et al., 1952; Flickinger e Patterson, 1967; Ware, 2007).

No IAM esperam-se manifestações clínicas de dor, alterações ao ECG, como desnivelamento do segmento ST, elevação da onda $\mathrm{T}$ e arritmias ventriculares algumas vezes fatais, aumento das enzimas cardíacas (Roe et al., 1977; Kaneko et al., 1997); ao ECO observa-se, muitas vezes, dilatação do coração como resultado do remodelamento cardíaco após o IAM (Topol et al., 1998) e aumento do índice de desempenho do miocárdio (Ling et al., 2003; Jegger et al.,
2006). Os objetivos deste trabalho foram avaliar as características clínicas, ECG, EC, ECO, bem como o perfil das enzimas creatina quinase (CK) e creatina quinase fração $\mathrm{MB}(\mathrm{CK}-\mathrm{MB})$ em cães portadores de pequeno infarto experimental agudo do miocárdio, e avaliar as arritmias após a injeção intramiocárdica por ECG e EC.

\section{MATERIAL E MÉTODOS}

Foram selecionados por meio de exames clínico, ECO, ECG, pressórico por método Doppler e exames hematológicos (hemograma, contagem plaquetária, pesquisa de anticorpos antileishmania, bioquímico completo, CK e CKMB), seis cães machos, sem raça definida, com idade entre um e cinco anos, e massa corporal de $10,95 \pm 3,4 \mathrm{~kg}$, os quais passaram por período de adaptação de um mês e eram provenientes do Centro de Controle de Zoonoses e Endemias de Betim, MG. Este experimento foi aprovado pelo Comitê de Ética da Universidade Federal de Minas Gerais, sob o protocolo n ${ }^{\circ} 133 / 2007$.

Os animais receberam cefalexina $20 \mathrm{mg} / \mathrm{kg}$, endovenosa (IV), 30 minutos antes do procedimento cirúrgico; aplicou-se, ainda, medicação pré-anestésica - morfina $0,3 \mathrm{mg} / \mathrm{kg}$ e tramal $2 \mathrm{mg} / \mathrm{kg}-$, por via intramuscular. A indução anestésica foi realizada com propofol, $4 \mathrm{mg} / \mathrm{kg}, \mathrm{IV}, 10$ minutos após a medicação préanestésica. A manutenção anestésica foi efetuada com isoflurano.

Com o animal anestesiado, foram realizadas duas toracotomias intervaladas de sete dias em cada cão, portanto todos os animais utilizaram colar elisabetano durante 15 dias, a partir da data da primeira toracotomia. $\mathrm{Na}$ primeira toracotomia, o IAM foi induzido por oclusão com fio poliglecaprone $25 \quad 3-0$ da artéria coronária descendente anterior (CAD) de acordo com Eyster (1993) (dia zero). Na segunda toracotomia - dia sete -, realizaram-se cinco injeções intramiocárdicas de $0,04 \mathrm{~mL}$ cada, com solução de cloreto de sódio 0,9\%, em diferentes pontos na área infartada visualizada macroscopicamente. Como procedimento analgésico, todos os animais receberam $0,1 \mathrm{mg} / \mathrm{kg}$ de meloxicam, via oral, a cada 24 horas, do dia zero ao quinto e do sétimo ao $13^{\circ}$ dia. Nas primeiras 96 horas após a realização de cada toracotomia, os animais foram 


\section{Cavalcanti et al.}

avaliados clinicamente a cada quatro horas, e os dados eram anotados em fichas individuais.

Como exames de acompanhamento, foram realizados ECG, EC, ECO e avaliação sanguínea. O ECG (Ecafix ECG40®), em 10 derivações, e o EC (Cardio flash plus $®)$ foram realizados na triagem e nos dias um (após a indução do infarto), sete (após a injeção intramiocárdica) e 69 do experimento (Tilley, 1992). Ainda, durante o exame de EC, os cães foram submetidos a caminhadas leves durante 20 minutos, a cada seis horas.

O acompanhamento por ECO, com aparelho Sonos 100CF® da Hewlett Packard, foi realizado nos dias cinco, 17, 34, 51 e 69. Os exames ecocardiográficos foram gravados em fitas de vídeocassete para avaliações posteriores.

No modo $\mathrm{M}$ realizaram-se as medidas de acordo com as recomendações de Lombard (1984), sendo que, ao final da diástole, foram obtidas as medidas de dimensão interna do ventrículo esquerdo (DDVE) e a espessura da parede septal do VE (SVEd) e da parede posterior do ventrículo esquerdo (PPd). Foram medidas, no final da sístole: dimensão interna do ventrículo esquerdo (DSVE); espessura da parede septal do VE (SVEs) e da parede posterior do ventrículo esquerdo (PPs).

Após a obtenção dessas medidas, foram calculadas, segundo Ware (2007), a porcentagem de encurtamento sistólico do diâmetro ventricular esquerdo (\%AD), a fração de ejeção (FE), as porcentagens de espessamento sistólico da parede posterior do VE (\%PP) e da parede septal do VE (\%PS) e as relações PPd:DDVE e SVEd:PPd.

No modo bidimensional (2D), foram avaliados o volume diastólico final do VE (VDf), o volume sistólico final do VE (VSf) e a fração de ejeção pelo método de Simpson (FES) biplanar, no corte 2 câmaras apical (Silva et al., 2003), além das medidas de átrio esquerdo (AE) e da raiz aórtica (Ao), de acordo com Rishniw e Erb (2000). Posteriormente, foi calculada a relação $\mathrm{AE}$ e Ao (AE:Ao) de cada exame.

No modo Doppler, foram avaliados o índice de desempenho miocárdico (IDM), a medida de velocidade das ondas E e A mitrais, e o tempo de ejeção (TE) integral do fluxo da via de saída do ventrículo esquerdo, e foi analisado o tempo de desaceleração mitral (TDE) (Ware, 2007).

Os mesmos exames de sangue realizados na triagem foram repetidos nos dias 30 e 60 . Além disso, nos três primeiros dias após a indução do IAM, a mensuração de CK e de CK-MB foi realizada a cada seis horas, e ambas as enzimas foram novamente medidas no dia sete. No dia 70, todos os animais foram sacrificados, sendo a área infartada avaliada em porcentagem da área do ventrículo esquerdo por programa computacional (Ps Adobe ${ }^{\circledR}$ Photoshop CS3 extended).

Os dados quantitativos SVEd, SVEs, PPd, PPs, \%PP, \%PS, PPd:DDVE, SVEd:PPd, DDVE, DSVE, FE, \%AD, VDf, VSf, FES, Em, Am, relação entre os picos de velocidade das ondas de enchimento do VE (E/Am), TDE, TE, IDM, AE, Ao, AE/Ao, TVI, CK e CK-MB apresentaram-se normais ao teste Kolmogorov-Smirnov, sendo realizada a análise de variação e usado o teste Dunnett a 5\% para comparações de médias, com os valores obtidos na triagem como referência.

Foram descritos e comparados pelo teste Tukey 5\%, após realização da análise de variação, as médias e os desvios-padrão dos infradesnivelamentos de ST ao EC nos diferentes dias, e foram descritos as médias e os números mínimos e máximos das arritmias ventriculares observadas ao EC.

\section{RESULTADOS E DISCUSSÃO}

Embora haja relatos de infarto em cães, há escassez na literatura da avaliação dessa afecção; além disso, não foram encontrados trabalhos de avaliação por EC da injeção no miocárdio, que é uma possível via terapêutica cardíaca para a utilização da terapia celular. Portanto, realizou-se este estudo com a finalidade de que importantes dados sejam fornecidos sobre uma cardiopatia que tem levado a muitas investigações no meio científico (Jegger et al., 2006).

Os seres humanos com IAM apresentam dor intensa e opressiva no peito, a qual irradia para o braço esquerdo, além de dispepsia, náuseas e vômitos (Topol et al., 1998), sendo que o vômito ocorre mais em infartos de parede inferior e anterior (Ingram et al., 1980; Fuller et al., 2009). 
Os sinais clínicos observados - apatia extrema, hiporexia de grande duração, sialorreia e vocalização - são compatíveis com os sinais clínicos de manifestação de dor comumente apresentados pelos seres humanos. Nas primeiras 24 horas, após indução do IAM, todos os animais apresentaram apatia intensa $(n=6)$, e metade vocalizou $(n=3)$, o que pode ser devido à dor no peito. Acredita-se que a sialorreia apresentada pelos animais $(n=6)$ seja devido a náuseas, mesmo que nenhum animal tenha apresentado vômito. Sabe-se que a sialorreia é um sinal de desconforto gástrico/entérico, podendo ser um equivalente aos sintomas dispépticos observados em humanos (Elwood et al., 2010). A hiporexia $(n=3)$ apresentada após a indução do IAM pode ser devido à dor ou à dispepsia e ela cessou em 72 horas. No pós-operatório da segunda cirurgia - sete dias após a realização da primeira cirurgia -, os animais mostraram-se muito mais alertas e sem apresentar vocalização, apatia extrema, sialorreia e hiporexia, o que indica que os sinais clínicos aqui expostos sejam semelhantes aos descritos em seres humanos com IAM.

Nenhum animal deste estudo apresentou insuficiência cardíaca congestiva nem veio a óbito devido às arritmias do pós-infarto imediato, mesmo sem receber terapia específica. Sabe-se que as arritmias ventriculares, principalmente a taquicardia ventricular (TV), causam cerca de $60 \%$ das mortes na primeira hora após o IAM (Topol et al., 1998). Todos os animais do presente experimento apresentaram TV nas primeiras 24 horas após IAM (Tab. 1). Hubka et al. (2006), ao estudarem o IAM em cães por EC no terceiro dia após o IAM, encontraram extrassístoles ventriculares em menor quantidade - mínimo 0 e máximo de 4789 episódios durante oito horas - que no presente estudo. Isso pode ser atribuído ao fato de o EC ter sido utilizado após o momento de maior frequência das arritmias ventriculares e pela menor duração dos exames realizados por aqueles autores.

Tabela 1. Frequência média (mínima - máxima) das arritmias ventriculares encontradas nos cães, nos diferentes momentos de avaliação, por eletrocardiografia contínua.

\begin{tabular}{ccccc}
\hline & CVPi & CV em Bigeminismo & CV pareados & TV \\
\hline Triagem & 0 & 0 & 0 & 0 \\
$1 \mathrm{~d}$ & $4196(90-13371)$ & $333,33(0-1257)$ & $2551(0-8955)$ & $7733(0-15270)$ \\
$7 \mathrm{~d}$ & $80,5(0-477)$ & $13(0-78)$ & $0,17(0-1)$ & 0 \\
$69 \mathrm{~d}$ & 0 & 0 & 0 & 0 \\
\hline
\end{tabular}

CVPi- Complexo ventricular prematuro isolado; CV- complexo ventricular; TV- taquicardia ventricular. 1d: primeiras 24 horas após a indução do infarto; 7d: primeiras 24 horas após a injeção intramiocárdica; 69d: 69 dias após a indução do infarto do miocárdio.

Em um estudo retrospectivo de IM em pequenos animais, observou-se que cinco cães que vieram a óbito súbito e inexplicável apresentaram TV ao ECG, sendo o IAM posteriormente confirmado (Driehuys et al., 1998). Dessa forma, deve-se assistir a cães com IAM e arritmias ventriculares graves.

No presente experimento, $67 \%$ dos animais apresentaram TV, sendo que entre 2 e $12 \%$ dessa arritmia ocorreram de forma sustentada, não havendo ocorrência de TV após a injeção intramiocárdica, o que corroba com o estudo de Tondato e colaboradores (2011), que não encontraram maior probabilidade da ocorrência de TV após a injeção intramiocárdica em porcos infartados experimentalmente. As arritmias ventriculares observadas estão ilustradas na Tab. 1 .
Importante mostrar que, ao ECG realizado no momento do infarto, dia um, e no dia da injeção intramiocárdica, dia sete, mostrou-se apenas CVP em um animal no dia um, o que não refletiu a gravidade das arritmias que ocorreram durante a monitoração por EC em 24 horas. Este resultado poderia ser observado, pois o ECG acompanha apenas $0,2 \%$ das 24 horas de que um dia é composto, e o animal é contido em cima de uma mesa (Cavalcanti et al., 2007).

Além das arritmias, observaram-se aumento de onda $\mathrm{T}$ em quatro animais e supradesnivelamento de ST em seis, ao ECG deste estudo. De acordo com a literatura, sabe-se que o aumento da onda $\mathrm{T}$ devido ao IAM em cães ocorre apenas nas primeiras horas após o evento isquêmico e que o aumento ventricular também pode causar aumento dessa onda (Tilley, 1992). Apenas os cães 1 e 5 apresentaram aumento dessa onda no 


\section{Cavalcanti et al.}

dia um - dia do infarto -, sendo que o cão 5 apresentou esse aumento também no dia sete, portanto, nos cães 1 e 5 o aumento dessa onda pode ser atribuído ao infarto e ao procedimento anestésico. Já nos outros dois cães - 3 e $4-$, o aumento da onda $\mathrm{T}$ ocorreu no dia 69 , o que sugere aumento cardíaco, principalmente quando associado ao aumento de duração do complexo QRS, como visto no cão 4.

Além das arritmias ventriculares, ocorreu supradesnivelamento de ST no ECG geralmente presente nas derivações pré-cordiais V1 e V2, no dia 69. Apenas um animal apresentou essa alteração em todos os momentos após o infarto, em duas derivações contíguas (V1 e V2); os outros animais só a apresentaram no dia 69. Sabe-se que a observação de alteração de ST depende da localização do infarto no VE e da porção do miocárdio acometido e que quanto mais ECG são realizados em um mesmo indivíduo, maior a constatação dessa alteração (Topol et al., 1998). No presente trabalho, os infartos provocados atingiram $13,4 \pm 5,2 \%$ da área total do ventrículo esquerdo e na região apical.

Em cães com IM, a observação de supradesnivelamento de ST ao ECG é pouco descrita. Na literatura pesquisada, apenas um cão em estudo retrospectivo (Liu et al., 1986) e um relato de caso (Almeida et al., 2007) evidenciaram essa alteração, ambas na derivação II do ECG. O presente estudo observou o supradesnivelamento nas derivações pré-cordiais V1 e V2, provavelmente em razão do tamanho e da localização do IAM, conforme Topol et al. (1998).

Quanto ao EC, o desnivelamento de ST foi aparentemente mais frequente que na avaliação ao ECG, ocorrendo em $67 \%$ dos animais no dia um, em $83 \%$ dos animais no dia sete e $67 \%$ dia 69 , contra 33,33 e $83 \%$, respectivamente, nos dias um, sete e 69 na avaliação por ECG. A frequência dessa alteração ocorreu sem diferença estatística na comparação entre os dias avaliados, provavelmente em razão da grande variação de apresentação em 24 horas (Tab. 2), sendo a mediana de apresentação de 7,5, 30 e de 12,5 episódios, para as avaliações dos dias um, sete e 69, respectivamente; portanto, aparentemente mais prevalente no dia sete. $\mathrm{O}$ fato de o EC ser mais sensível do que o ECG na identificação das alterações de ST é porque os exercício físico aumentam a identificação do infarto (Topol et al., 1998) e também porque os animais passeavam e passavam por situações de alta frequência cardíaca enquanto eram submetidos ao exame EC.

Tabela 2. Avaliação de infradesnivelamento de ST nos diferentes canais de Holter, em cães com infarto miocárdico

\begin{tabular}{ccccccc}
\hline & \multicolumn{2}{c}{$1 \mathrm{~d}$} & \multicolumn{2}{c}{$7 \mathrm{~d}$} & \multicolumn{2}{c}{$69 \mathrm{~d}$} \\
\cline { 2 - 7 } & $\begin{array}{c}\mathrm{N}^{\mathbf{0}} \text { de } \\
\text { episódios } \\
(\text { média } \pm \mathrm{DP})^{\mathbf{A}}\end{array}$ & $\begin{array}{c}\text { Depressão } \\
\text { máxima }\end{array}$ & $\begin{array}{c}\mathrm{N}^{\mathbf{0}} \text { de episódios } \\
(\text { média } \pm \mathrm{DP})^{\mathbf{A}}\end{array}$ & $\begin{array}{c}\text { Depressão } \\
\text { máxima }\end{array}$ & $\begin{array}{c}\mathrm{N}^{\mathbf{0}} \text { de } \\
\text { episódios } \\
(\text { média } \pm \mathrm{DP})^{\mathbf{A}}\end{array}$ & $\begin{array}{c}\text { Depressão } \\
\text { máxima }\end{array}$ \\
\hline $\mathrm{C} 1$ & $0 \pm 0$ & - & $7,5 \pm 8,38 \mathrm{~A}$ & $4,6 \mathrm{~mm}$ & $5,5 \pm 11,62 \mathrm{~A}$ & $3,8 \mathrm{~mm}$ \\
$\mathrm{C} 2$ & $2,88 \pm 4,44 \mathrm{~B}$ & $4,3 \mathrm{~mm}$ & $12,83 \pm 8,40 \mathrm{~B}$ & $4,2 \mathrm{~mm}$ & $9 \pm 9,51 \mathrm{~B}$ & $3,7 \mathrm{~mm}$ \\
$\mathrm{C} 3$ & $6,66 \pm 5,57 \mathrm{C}$ & $4,5 \mathrm{~mm}$ & $13,67 \pm 8,98 \mathrm{C}$ & $4 \mathrm{~mm}$ & $7,83 \pm 8,28 \mathrm{C}$ & $3,4 \mathrm{~mm}$ \\
\hline
\end{tabular}

C1: canal 1; C2: canal 2; C3: canal 3; 1d: primeiras 24 horas após a indução do infarto; 7d: primeiras 24 horas após a injeção intramiocárdica; 69d: 69 dias após a indução do infarto do miocárdio; $\mathrm{N}^{\circ}$ : número; DP: desvio padrão.

*Letras diferentes na mesma linha representam diferença estatística ao Teste Tukey $5 \%$.

Neste estudo, as situações nas quais foi mais detectado infradesnivelamento de ST foram no momento de colocação do aparelho e quando os cães passeavam. Para evitar danificações no aparelho de EC, os animais receberam, durante o exame, colar restritivo elizabetano, o que causou agitação nos animais nos dias um e 69. Todavia, Yamada e Tokuriki (2000) e Cavalcanti et al. (2007) demonstraram que não houve diferença na FC média avaliada por EC entre os cães que utilizaram e os que não utilizaram o colar elizabetano, e isso provavelmente ocorreu em função de o tempo de análise (24 horas) ocultar a agitação inicial provocada pela colocação do colar de restrição. No exame realizado no dia sete, os cães já estavam com colar restritivo; assim, é muito provável que a manipulação do animal no momento da colocação do aparelho cause nervosismo e, consequentemente, desníveis de ST. Além do momento de colocação 
do aparelho, quando os animais passeavam, eles se exercitavam e tinham contato com outras espécies animais, o que aumentava a FC, por isso os muitos momentos de FC alta e de alteração de ST ocorriam também nos horários de passeio anotados nas fichas individuais dos cães.

Houve aumento significativo nos índices ECO VDf, VSf, VDVE e VSVE avaliados pelo método de Simpson e Teichholz (Tab. 3). O método de Simpson é mais vantajoso que o método de Teichholz, comumente utilizado na medicina veterinária, por ser de avaliação mais global da função ventricular (Topol et al., 1998). O diâmetro aumentado das câmaras cardíacas induz ao aumento na espessura das paredes livre e septal, caracterizando a hipertrofia excêntrica. Além disso, também ocorre aumento da função sistólica da parede saudável na tentativa de compensar a falha segmentar funcional (Jegger et al., 2006).

Tabela 3. Valores médios dos parâmetros ecocardiográficos avaliados em diferentes momentos, em cães infartados experimentalmente

\begin{tabular}{|c|c|c|c|c|c|c|}
\hline & Triagem & $5 \mathrm{~d}$ & $17 d$ & $34 d$ & $51 d$ & $69 d$ \\
\hline SVEd & 673,3 & 702,8 & 677,7 & 642,7 & 644,7 & 646,2 \\
\hline SVEs & 896,2 & 913,0 & 880,2 & 889,2 & 888,0 & 942,7 \\
\hline PPd & 724,3 & 662,8 & 693,8 & 670,2 & 652,0 & 688,2 \\
\hline PPS & 982,3 & 886,0 & 903,8 & 932,5 & 882,3 & 923,3 \\
\hline$\% \mathrm{PP}$ & 36,16 & 33,73 & 31,30 & 40,78 & 36,67 & 34,95 \\
\hline$\% \mathrm{PS}$ & 33,98 & 30,01 & 30,28 & 40,94 & 45,73 & 44,88 \\
\hline $\mathrm{PPd} / \mathrm{DDVE}$ & 0,238 & 0,208 & 0,225 & 0,210 & 0,198 & $0,197 *$ \\
\hline $\mathrm{SVEd} / \mathrm{PPd}$ & 0,935 & 1,06 & 0,978 & 0,967 & 0,980 & 0,945 \\
\hline DDVE & 30,43 & 32,35 & 32,22 & 32,27 & $34,05^{*}$ & $35,02 * *$ \\
\hline DSVE & 17,70 & 19,25 & 19,35 & 18,78 & $21,48 *$ & $21,63^{*}$ \\
\hline $\mathrm{FE}$ & 73,83 & 72,17 & 71,55 & 71,68 & 67,22 & 69,23 \\
\hline$\% \mathrm{AD}$ & 41,55 & 40,17 & 39,70 & 40,15 & 36,78 & 38,42 \\
\hline VDf & 20,80 & 20,46 & 22,65 & 23,45 & 26,35 & $29,37 * *$ \\
\hline VSf & 6,03 & 6,24 & 6,20 & 6,62 & 7,64 & $8,72 * *$ \\
\hline FES & 71,17 & 67,03 & 71,52 & 71,30 & 70,13 & 70,88 \\
\hline TVI & 12,68 & 11,07 & 10,83 & 11,60 & 11,05 & 12,04 \\
\hline $\mathrm{Em}$ & 79,01 & 68,68 & 73,08 & 70,57 & 67,26 & 73,98 \\
\hline $\mathrm{AM}$ & 50,97 & 48,01 & 45,25 & 46,45 & 42,52 & 47,43 \\
\hline $\mathrm{E} / \mathrm{Am}$ & 1,59 & 1,46 & 1,62 & 1,53 & 1,60 & 1,56 \\
\hline TDE & 83,47 & 79,02 & 84,72 & 82,50 & 82,36 & 89,30 \\
\hline TE & 187,2 & 189,3 & 184,3 & 197,4 & 191,8 & 189,2 \\
\hline IDM & 0,20 & 0,25 & 0,26 & 0,24 & $0,40 * *$ & $0,37 *$ \\
\hline $\mathrm{AE}$ & 1,8 & 1,8 & 1,71 & 1,99 & 1,78 & 1,81 \\
\hline Ao & 1,46 & 1,46 & 1,48 & 1,47 & 1,52 & 1,55 \\
\hline AE/Ao & 1,22 & 1,27 & 1,16 & 1,42 & 1,23 & 1,25 \\
\hline
\end{tabular}

Xd: X dias após a indução do infarto miocárdico; SVEd: espessura da parede septal do ventrículo esquerdo (VE) na diástole (mm); SVEs: espessura da parede septal do VE na sístole $(\mathrm{mm})$; PPd: espessura da parede posterior do VE na diástole (mm); PPs: espessura da parede posterior do VE na sístole (mm); \%PP: porcentagem do espessamento sistólico da parede livre do VE; \%PP: porcentagem do espessamento sistólico da parede septal do VE; PPd/DDVE: relação da PPd e do diâmetro diastólico do VE; SVEd/PPd: relação do SVEd pela PPd; DDVE: diâmetro diastólico final do VE em mm; DSVE: diâmetro sistólico final de VE em mm; FE: fração de ejeção calculada pelo método de Teichholz em porcentagem; \%AD: fração de encurtamento da fibra miocárdica calculada pelo método de teichholz; VDf: volume diastólico final do VE em mL calculada pelo método de Simpson; VSf: volume sistólico final de VE em $\mathrm{mL}$ calculado pelo método de Simpson; FES: fração de ejeção calculado pelo método de Simpson em porcentagem; Em: pico da velocidade da onda E mitral $(\mathrm{cm} / \mathrm{s})$; Am: pico da velocidade da onda A mitral $(\mathrm{cm} / \mathrm{s})$; E/Am: relação das ondas E e A mitral; TDE: tempo de desaceleração da onda E mitral em ms; TE: tempo de ejeção; IDM: índice de desempenho miocárdico; $\mathrm{AE}$ : dimensão atrial esquerda ao modo $\mathrm{B}(\mathrm{mm})$; Ao: dimensão da raiz aórtica ao modo $\mathrm{B}$ (mm); AE/Ao: relação das dimensões entre AE e Ao; TVI: integral do fluxo da via de saída do ventrículo esquerdo (cm). Valores diferentes pelo teste de Dunnet a $5 \%\left(^{*}\right)$ e a $1 \%(* *)$ em comparação com a triagem. 


\section{Cavalcanti et al.}

Porém, no presente estudo, os índices SVEd, SVEs, PPd, PPs, \%PP, \%PS, \% AD, FE, FES não se alteraram (Tab. 3), sendo de conhecimento que a hipertrofia ventricular em seres humanos só ocorre em infartos que ocupam, no mínimo, $20 \%$ da área do VE e mais provável em áreas maiores que 30\% (Topol et al., 1998); portanto, é muito provável que as paredes do VE deste experimento não hipertrofiaram nem houve aumento da função sistólica em razão de o tempo experimental ser curto e de a área infartada média ser $13,4 \%$ da área do VE.

A cardiomiopatia dilatada é considerada a doença cardíaca mais comum de cães de raças grandes e cursa com diminuição dos índices da função sistólica (Ware, 2007). Entretanto, é possível que parte desses animais apresente arteriosclerose, principalmente do tipo hialina, acarretando isquemia, dilatação cardíaca, diminuição dos índices sistólicos e insuficiência cardíaca congestiva (Falk e Jönsson, 2000). Estudos em cães mostraram que $77 \%$ dos animais que exibiram IM pesavam mais de $15 \mathrm{~kg}$ (Falk e Jönsson, 2000) ou eram de raças grandes (Driehuys et al., 1998). Esses animais apresentaram pequenos infartos que culminaram com morte de origem cardíaca ou durante o procedimento anestésico (Falk e Jönsson, 2000).

$\mathrm{Na}$ avaliação da função diastólica pelo influxo mitral, apenas um animal, cinco dias após o IAM, apresentou disfunção e o TDE não se alterou. A disfunção diastólica constitui um importante marcador quando a função sistólica não diferencia o prognóstico dos pacientes, além de preceder a disfunção sistólica em muitas cardiopatias (Topol et al., 1998).

A baixa capacidade dos índices sistólicos e diastólicos na avaliação da disfunção ventricular após o IAM, em seres humanos, foi também relatada. O IDM é um índice que avalia as funções diastólicas e sistólicas ao mesmo tempo, sendo, muitas vezes, o único índice que aumenta nessa afecção (Ling et al., 2003; Jegger et al., 2006). No presente artigo, as alterações significativas encontradas ao ECO foram a dilatação ventricular esquerda e o aumento do IDM (Tab. 3).

Neste estudo, o IDM chegou a aumentar em $100 \%$ quando comparado com o valor observado na triagem. Os índices avaliados pelo método de Simpson aumentaram 41,2\% (VDf) e 44,6\% (VSf), enquanto o DDVE aumentou $15 \%$ e o DSVE 21\%, mostrando aumento maior do IDM, seguido dos índices propostos por Simpson. Estes resultados corroboram com a medicina humana, que utiliza o método Simpson em detrimento do método calculado pelo modo $\mathrm{M}$ na avaliação do remodelamento cardíaco no IM e da utilização do IDM em pacientes com coronariopatias (Topol et al., 1998; Jegger et al., 2006).

Outro índice que mostrou diminuição significativa foi o PPd/DDVE (Tab. 3), o que pode ter três explicações: 1- afilamento da parede livre na diástole ao longo do experimento; 2- dilatação diastólica da câmara ventricular esquerda ao longo do experimento; e 3- as possibilidades 1 e 2 ocorreram conjuntamente. Como mostrado na Tab. 3, a PPd não se afilou ao longo deste estudo, e o DDVE aumentou; logo, pode-se concluir que a possibilidade 2 é a mais concreta.

A creatina quinase $(\mathrm{CK})$ é uma enzima amplamente utilizada para determinação de doenças neuromusculares dos animais domésticos e apresenta a fração $\mathrm{MB}$, que é a subunidade específica do músculo cardíaco. Em humanos, a dosagem da CK-MB vem sendo utilizada como principal método para confirmação ou exclusão de infarto agudo do miocárdio (Roe et al., 1977).

Neste estudo, os valores de CK obtidos na triagem e de 66 horas em diante encontram-se dentro da margem de referência para cães - 20 $473 \mathrm{U} / \mathrm{l}$ - e adicionalmente foram os mesmos valores que se apresentaram estatisticamente iguais (Tab. 4), segundo Kaneko et al. (1997). 
Infarto agudo do miocárdio...

Tabela 4. Valores médios em U/L das análises obtidas de creatina quinase $(\mathrm{CK})$ e de creatina quinase fração MB (CK-MB) em diferentes momentos, em cães infartados experimentalmente

\begin{tabular}{ccc|ccc}
\hline Momento & CK & CK-MB & Momento & CK & CK-MB \\
\hline Triagem & 168,4 & 69,5 & $48 \mathrm{~h}$ & $1059,0^{*}$ & $375,3^{*}$ \\
$6 \mathrm{~h}$ & $2276,1^{* *}$ & $920,3^{* *}$ & $54 \mathrm{~h}$ & $726,7^{*}$ & 373,4 \\
$12 \mathrm{~h}$ & $2560,9^{* *}$ & $859,8^{* *}$ & $60 \mathrm{~h}$ & $483,7^{*}$ & 360,2 \\
$18 \mathrm{~h}$ & $2102,5^{* *}$ & $682,7^{* *}$ & $66 \mathrm{~h}$ & 366,9 & 343,4 \\
$24 \mathrm{~h}$ & $1732,18^{* *}$ & $599,6^{*}$ & $72 \mathrm{~h}$ & 298,4 & 225,1 \\
$30 \mathrm{~h}$ & $1685,7^{* *}$ & $603,0^{* *}$ & $7 \mathrm{~d}$ & 217,9 & 170 \\
$36 \mathrm{~h}$ & $1549,7^{* *}$ & $509,7^{*}$ & $30 \mathrm{~d}$ & 187,0 & 77,8 \\
$42 \mathrm{~h}$ & $1383,2^{* *}$ & $445,3^{*}$ & $60 \mathrm{~d}$ & 117,8 & 72,8 \\
\hline
\end{tabular}

Xh- X horas após a indução do infarto do IAM; Xd- X dias após o infarto miocárdico.

Valores diferentes pelo teste de Dunnet a $5 \%(*)$ e a $1 \%(* *)$ em comparação com a triagem.

Os valores de CK e de CK-MB variaram com sexo, lesão muscular, idade, atividade física e tamanho da área cardíaca infartada. Em estudo que avaliou as enzimas CK e CK-MB no IAM de cães, durante quatro dias, foram encontrados valores antes do evento para CK de 223IU/L (31 a 914) e para CK-MB 19,28IU/L (min 0 e max 76) (Roe et al., 1977). A dosagem seriada da CK e da CK-MB é fundamental para o diagnóstico de infarto, e os valores médios do atual experimento obtidos na triagem - $\mathrm{CK}$ igual a 168IU/L e CK-MB igual a 69,5IU/L - estão contidos na variação encontrada por Roe et al. (1977), porém há poucos trabalhos na literatura a respeito da dosagem de CK-MB em cães para se inferir comentários mais confiáveis.

No presente trabalho, observou-se que o pico de CK-MB foi em seis horas após o IAM, e o pico de CK em 12 horas. Em seres humanos, $45 \%$ dos indivíduos apresentam pico de CK-MB antes do CK total, entretanto o pico dessa enzima ocorre entre 10 e 24 horas após o evento isquêmico (Topol et al., 1998). Portanto, é provável que os cães apresentem o pico de CK-MB antes que o observado em seres humanos, porém novos estudos nessa espécie são necessários para melhores conclusões.

Os resultados anátomo-histopatológicos, aos 70 dias de estudo, confirmaram o infarto por visualização de área externa brancacenta menos rígida em região apical do VE. Nos cortes longitudinais dessa mesma parede ventricular, observaram-se afilamento e palidez da região apical, e ao exame histológico constataram-se morte das células musculares e substituição do tecido contrátil por tecido colagênico; alterações essas que, de acordo com Pereira (2006), são observadas no infarto do miocárdio.

\section{CONCLUSÕES}

Este estudo mostra relevantes dados sobre o infarto do miocárdio, melhorando a identificação dessa afecção em cães, seja na forma de infarto intramural microscópico, seja secundário à obstrução de vaso coronário importante.

\section{AGRADECIMENTOS}

Os autores agradecem à Fundação de Amparo à Pesquisa do Estado de Minas Gerais (FAPEMIG), por financiar este experimento; à Agener União, por disponibilizar medicamentos; à Royal Canin, por fornecer ração aos animais; e ao Conselho Nacional de Desenvolvimento Científico e Tecnológico (CNPq), por fornecer bolsa de doutorado ao aluno G.A.O. Cavalcanti.

\section{REFERÊNCIAS}

ALMEIDA, G.L.G.; TORTELLY, R.; SOARES, J.R.F. et al. Relato de caso de infarto agudo do miocárdio em cão. Rev. Univ. Rural-Série Ciências da Vida, v.27, supl., p.521-523, 2007.

CAVALCANTI, G.A.O.; NOGUEIRA, R.B.; GONÇALVES, R.S. et al. Influência da utilização de colar elizabetano ou colar cervical em cães submetidos à Eletrocardiografia Contínua (HOLTER). Rev. Univ. Rural - Série Ciências da Vida, v. 27, supl., p.461463, 2007.

DRIEHUYS, S.; VAN WINKLE, T.J.; SAMMARCO, C.D. et al. Myocardial infarction in dogs and cats: 37 cases (1985-1994). J. Amer. Vet. Med. Associat., v.213, p.1444-1448, 1998.

ELWOOD, C.; DEVAUCHELLE, P.; ELLIOTT, J. et al. Emesis in Dogs: a review. The J. Small Anim. Pract., v.51, p.4-22, 2010. 


\section{Cavalcanti et al.}

EYSTER, G.E. Cardiovascular System. In: SLATTER, D. Textbook of Small Animal Animal Surgery. 2.ed. Pennsylvania: Saunders Company, 1993. p.820-929.

FALK, T.; JONSSON, L. Ischemic heart disease in the dog. J. Small Anim.Pract., v.41, p.97-103, 2000.

FLICKINGER, G.L.; PATTERSON, D.F. Coronary lesions associated with congenital subaortic stenosis in the dog. J. Pathol. Bact., v.4, p.133-140, 1967.

FULLER, E.E.; ALEMU, R.; HARPER, J.F. et al. Relation of nausea and vomiting in acute myocardial infarction to location of the infarct. Amer. J. Card., v.12, p.138-140, 2009.

HUBKA, P.; BERNADIC, M.; HULÍN, I. Long-term monitoring of the changes in signal-averaged ecg after coronary artery occlusion and intracoronary endothelin-1 injection in dogs. Physiol. Res., v.55, p.914, 2006.

INGRAM, D.A.; FULTON, R.A.; PORTAL, R.W. et al. Vomiting as a diagnostic aid in acute ischaemic cardiac pain. Br. Med. J., v.281, p.636-637, 1980.

JEGGER, D.; JEANRENAUD, X.; NASRATULLAH, M. et al. Noninvasive Doppler-derived myocardial performance index in rats with myocardial infarction: validation and correlation by conductance catheter. Am. J. Physiol. - Heart and Circulatory Physiology, v.290, p.1540-1548, 2006.

KANEKO, J.J.; HERVEY, J.W.; BRUSS, M.L. Appendixes. In: KANEKO, J.J.; HARVEY, J.W.; BRUSS, M.L. Clinical Biochemistry of Domestic Animals. 5.ed. San Diego:Academic Press, 1997. p.885-905.

LERI, A.; ANVERSA, P.; FRISHMAN, W.H Cardiovascular regeneration and stem cell therapy. 1.ed. Massachusetts: Blackwell Publishing, 2007. 229p.

LI, Y.; YAO, Y., SHENG, Z. et al. Dual-modal tracking of transplanted mesenchymal stem cells after myocardial infarction. Int. J. Nanotecnol., v.6, p.815823, 2011.

LINDSAY, S.; CHAIKOFF, I.L.; GILMORE J.W. Arteriosclerosis in the dog. I. Spontaneous lesions in the aorta and the coronary arteries. A.M.A. Arch. Pathol., v.53, p.281-300, 1952.

LING, L.H.; TEI, C.; MCCULLY, R.B. et al. Analysis of systolic and diastolic time intervals during dobutamine-atropine stress echocardiography: diagnostic potential of the Doppler myocardial performance index. J. Am. Soc. Echocardiography, v.14, p.978-986, 2003.
LIU, S.K.; TILLEY, L.P.; TAPPE, J.P. et al. Clinical and pathologic findings in dogs with atherosclerosis: 21 cases (1970-1983). J. Am. Vet. Med. Assoc., v.189, p.227-232, 1986.

LOMBARD, C.W. Normal values of the canine Mmode echocardiogram. Am. J. Vet. Res., v.45, p.20152018, 1984.

PEREIRA, F.E.L. Degenerações, morte celular, alterações do interstício. In: FILHO, G. B. Bogliolo Patologia.7 ed. Rio de Janeiro: Guanabara Koogan, 2006. p. 43-82.

RISHNIW, M.; ERB, H.N. Evaluation of four 2dimensional echocardiographic methods of assessing left atrial size in dogs. J. Vet. Inter. Med., v.14, p. 429$435,2000$.

ROE, C.R.; COBB, F.R.; STRAMER, C.F. The relationship between enzymatic and histologic estimates of the extent of myocardial infarction in conscious dogs with permanent coronary occlusion. Circulation, v.55, p.438-449, 1977.

SILVA, C.E.S.S.; FERREIRA, L.D.C.; MONACO, C.G. et al. O ecocardiograma no apoio à decisão clínica. 3.ed. Rio de Janeiro: Livraria e Editora Revinter, 2003, 274p.

TILLEY, L.P. Essentials of canine and feline electrocardiography. 3.ed. Philadelphia: Lea \& Febiger, 1992. 470p.

TONDATO, F.; ROBINSON, K.; CUI, J. et al. Effects on arrhythmogenesis and arrhythmic threshold of injection of autologous fibroblasts into myocardial infarcts in adult pigs. J. Cardiov. Transplant. Res. No Prelo. DOI: 10.1007/s12265-011-9316-9.

TOPOL, E.J.; CALIFF, R.M.; ISNER, J.M. et al. Textbook of Cardiovascular Medicine. Philadelphia: Lippincott-Raven, 1998. 2732p.

YAMADA, M.; TOKURIKI, M. Effects of a canine Elizabethan collar on ambulatory electrocardiogram recorded by a holter recording system and spontaneous activities measured continuously by an accelerometer in beagle dogs. J. Vet. Med. Sci., v.62, p.549-552, 2000.

ZHANG, D.Z.; GAI, L.Y.; HONG-WEI, L. et al. Transplantation of autologous adipose-derived stem cells ameliorates cardiac function in rabbits with myocardial infarction. Chin. Med. J., v.120, p.300307, 2007.

WARE, W.A. Cardiovascular disease in small animal medicine. 1.ed. London: Manson Publishing, 2007. 396p. 